5. G. W. Mackey, Borel structure in groups and their duals, Trans. Amer. Math. Soc. 85 (1957), 134-165.

6. J. T. Oxtoby and S. M. Ulam, On the existence of a measure invariant under a transformation, Ann. of Math. 40 (1939), 560-566.

7. V. N. Sudakov, Linear sets with quasi-invariant measure, Dokl. Akad. Nauk SSSR 127 (1959), 524-525. (Russian)

8. Y. Umemara, Measures on infinite dimensional vector spaces, dittoed seminar notes from Kyoto University.

9. A. Weil, L'intégration dans les groupes topologiques et ses applications, Actualités Sci. Ind., No. 869, Hermann, Paris, 1940.

Berkeley, California

\title{
A NOTE ON THE RECURSIVE UNSOLVABILITY OF PRIMITIVE RECURSIVE ARITHMETIC
}

\author{
RICHARD E. GRANDY ${ }^{1}$
}

We wish to show the recursive unsolvability of primitive recursive arithmetic (PRA). By PRA we mean a quantifier-free formal system of arithmetic which has expressions for all primitive recursive functions. In such a system all valid variable free formulas are provable and both of the Gödel incompleteness theorems hold. Further, we may define in the system bounded quantifiers and (for a suitable Gödel numbering) the following primitive recursive functions: th $(x)$, a function which enumerates the Gödel numbers of theorems of PRA, and $\operatorname{sub}(n, m)$, the function whose value is the Gödel number of the formula obtained by replacing the first variable in alphabetic order by the numeral $n$ through the formula number $m .^{2}$

If there is a recursive decision procedure for PRA, then the set of Gödel numbers of nontheorems is recursively enumerable. But if a set is recursively enumerable then it is primitive recursively enumerable. Thus if PRA is solvable there is a primitive recursive function whose range is precisely the set of Gödel numbers of nontheorems.

Assume there exists such a function $f$. Consider the formula

$$
\operatorname{th}(x)=\operatorname{sub}\left(x_{0}, x_{0}\right) \supset(E z) . z \leqq x \& f(z)=\operatorname{sub}\left(x_{0}, x_{0}\right) .
$$

Received by the editors March 15, 1965.

1 The author is indebted to J. R. Guard for his encouragement and to the National Science Foundation for financial support.

2 Detailed proofs may be found in J. R. Guard, The independence of transfinite induction up to $\omega^{\omega}$ in recursive arithmetic, unpublished dissertation, Princeton University, 1962, or H. E. Rose, On the consistency and undecidability of recursive arithmetic, Z. Math. Logik Grundlagen Math. 7 (1961), 124-135. 
Let the Gödel number of this formula be $i$, then the formula whose Gödel number is $\operatorname{sub}(i, i)$ will be

$$
\operatorname{th}(x)=\operatorname{sub}(i, i) \supset(E z) . z \leqq x \& f(z)=\operatorname{sub}(i, i) .
$$

Suppose this formula is provable. There must be some $k$ such that $\operatorname{th}(k)=\operatorname{sub}(i, i)$ is valid and hence provable. But if this formula and (2) are provable then by modus ponens and substitution (Ez). $z \leqq k \& f(z)=\operatorname{sub}(i, i)$. Thus $\operatorname{sub}(i, i)$ is one of the first $k$ nontheorems, contrary to hypothesis.

Suppose (2) is not a theorem. By hypothesis, $f$ enumerates all nontheorems, so there must be some $n$ such that $f(n)=\operatorname{sub}(i, i)$. But now we may obtain a proof of (2) as follows: For each number $m$ less than $n, \operatorname{th}(m) \neq \operatorname{sub}(i, i)$ will be provable, thus the conjunction of these $n$ formulas will be provable. But this gives $\operatorname{th}(x) \neq \operatorname{sub}(i, i) \bigvee n \leqq x$, and hence $\operatorname{th}(x) \neq \operatorname{sub}(i, i) \bigvee n \leqq x \& f(n)=\operatorname{sub}(i, i)$, from which (2) follows immediately.

Thus the formula (2) is neither provable nor unprovable, so there must be no such formula and PRA is unsolvable.

Princeton University 